\title{
Herb lotions to regrow hair in patients with intractable alopecia areata
}

\author{
Hideo Nakayama*, Ko-Ron Chen \\ Meguro Chen Dermatology Clinic, Tokyo, Japan
}

\begin{abstract}
The history of herbal medicine in China goes back more than 1,000 years. Many kinds of mixtures of herbs that are effective to diseases or symptoms have been transmitted from the middle ages to today under names such as Traditional Chinese Medicine (TCM) in China and Kampo in Japan.

For the treatment of severe and intractable alopecia areata, such as alopecia universalis, totalis, diffusa etc., herb lotions are known to be effective in hair regrowth. Laiso ${ }^{\circledR}$, Fukisin ${ }^{\circledR}$ in Japan and $101^{\circledR}$ in China are such effective examples. As to treat such cases, systemic usage of corticosteroid hormones are surely effective, however, considering their side effects, long term usage should be refrained. There are also these who should refrain such as small children, and patients with peptic ulcers, chronic infections and osteoporosis. AL-8 and AL-4 were the prescriptions removing herbs which are not allowed in Japanese Pharmacological regulations from 101, and salvia miltiorrhiza radix (SMR) is the most effective herb for hair growth, also the causation to produce contact sensitization. Therefore, the mechanism of hair growth of these herb lotions in which the rate of effectiveness was in average $64.8 \%$ on 54 severe intractable cases of alopecia areata, was very similar to DNCB and SADBE.

The most recommended way of developing herb lotion with high ability of hairgrowth is to use SMR but its concentration should not exceed $2 \%$, and when sensitization occurs, the lotion should be changed to Laiso ${ }^{\circledR}$ or Fukisin ${ }^{\circledR}$, which do not contain SMR.

Relapse of severe alopecia areata after or during hairgrowth is quite common, therefore, to maintain the cured conditions, the allergens to break immune priviledge (IP), such as metal allergy and / or atopic mite allergy should be investigated. When these allergens were removed, the cured conditions were maintained on an average of 4 years. It should also be noted that such allergen control (in other terms allergen avoidance or allergen elimination) should be performed, along with antisymptomatic treatments consisted of herb lotion application and temporary systemic usage of corticosteroid hormones.
\end{abstract}

\section{Introduction -A brief history of herb medicine}

\section{Herbs in the ancient time}

Many diseases were already present when the history of mankind started. Medical care had been necessary even in ancient times when medical science was not present. Besides religious prayer commonly performed all around the world, primitive surgery started in India, and various plant components were evaluated as drugs in China and Greek. In $\mathrm{AD} 77$, Dioscorides, the medical doctor of the notorious emperor Nero, wrote a book entitled "Greek Herbs" which described 958 species of herbs in Rome [1]. Around AD 200, Hwa Toh, a Chinese "Ishan" (medical doctor) invented anesthesia using boiled hemp, Ma Hwe San, a kind of marihuana mixed in rice wine, to induce sleep before his surgical operation was made. That is why later anesthesia had been called Ma Zwei, "hemp-induced intoxication", for many years in China and Japan, and the term has been retained even after the introduction of ether and chloroform in the 19th century [2].

In AD 610, Chinese medical textbooks called "Zhu Pyng Eng Hoh Rung" (Descriptions of various diseases) were written and published by the order of the emperor of Swe Dynasty. The author was Zha Eng Huan, and amazingly, some names of diseases and symptoms in the books are the same as those used today, such as dysentery, comedo, rosacea, eczema, nasal bleeding, erysipelas, carbuncle, sarcoma, ulcer, furuncle, fistula, hemorrhoid, psoriasis, tinea, scabies, rhus dermatitis, rabies, hornet sting, bone fracture, fluor, amenorrhea, hemoptosis, pregnancy, constipation, icterus, warts, etc. [3-4].
In China many kinds of herbs were usually used in mixed together, and the best and effective combinations were passed down from generation to generation. Publications on such herbal combinations included "I Shin Huan" (Medical prescriptions) published in AD 982, and "Wai ko Zheng Zhong" (Textbook of Surgery) written by Chen Su Kong in AD 1617, etc. The biggest medical books "Peng Zhao Zheng" (Herb Items) were written by Li Suh Zheng. He spent 27 years to write 52 volumes describing a total of 1892 herbs. These huge accumulation of Chinese herbs for the treatment of various diseases was published in 1593 in Nanjing.

It is important to note that those effective and reliable formulas were retained and published, whereas those which were deemed ineffective by later generations were abandoned and not transmitted to later publications. Therefore, such historical selection had been performed for more than 1,000 years.

\section{Good herbs were passed down}

The famed "Zhu Pyng Eng Hah Rung" (Descriptions of various diseases) having 50 volumes were imported to Japan via Korea in the

Correspondence to: Hideo Nakayama, Meguro Chen Dermatology Clinic Shinyo-CK Building 6F 3-3-5, Kami-Ohsaki, Shinagawa-ku, Tokyo 141-0021, Japan, Tel: +81-3-3786-1678; E-mail: nakayamadermatology@eos.ocn.ne.jp

Key words: herb lotion, Salivia miltiorrhiza, alopecia areata, metal allergy, atopic alopecia

Received: May 22, 2017; Accepted: June 19, 2017; Published: June 22, 2017 
7th Century. In 1528, Sozen Asano translated 24 volumes of Chinese medical books "I Such Da Zhen" (Medical Textbook) written by Xiong jun [2]. The Japanese government at that time, Tokugawa, made a wide herb field in the center of Edo (now Tokyo), so that the usage of Chinese herbal medicine would be widely used in Japan. A hospital using herbal opened at the same time in the center of Edo.

In Europe, anatomy started in Italy in 1315, and it was permitted in general by 1377 [1]. It urged the progress of surgery and general medicine, and the invention of the microscope in 1590 opened the gate for modern medicine. Effective chemicals from herbs were discovered one by one, such as aspirin from the willow bark in 1897 by Hoffman, ephedrine from Ma Hua (Ephedrs herb) in 1885 by Nagai, quinine from quina, glycyrrhetinic acid from glycyrrhizae radix, reserpine from lauwolfia serpengina, etc.

Such herbal medicine has been kept after the excellent European and American medicine was introduced in 19th Century. In China the term Traditional Chinese Medicine (TCM) has been used, while in Japan, term Kampo (Chinese medicine) has been used for many years, and they are still used today.

The merit of these traditional medicines is that generally as the several herbs are used as combinations, various actions are also taken to produce good results of treatment, and the quenching of drugs can ameliorate the adverse effects of each component. The effects have been demonstrated through evaluations of later generations, however, double blind tests have not been performed except for several herb medications.

In both countries, evaluating a long history of usage and treatment, a number of herbs have been agreed for medical usage today, omitting ordinary three phase trials of basic and clinical evaluation, because serious side effects have been rare with these traditional herbs.

Side effects of perorally administered 52 types of mixed Chinese herbal medicine studied in Japan in the recent 34 years from 1980 to 2014 showed five cases of toxic epidermal necrolysis (TEN), among which one case caused by Dajian Zhong Tang to enhance the intestinal movement after abdominal surgery was fatal. Others were caused by all different various herbs. Further cases included each one case of Stevens Johnson Syndrome (SJS) and Drug-induced hypersensitivity syndrome (DIHS), five cases of generalized dermatitis, and three cases of anaphylactic shock [5]. The incidence has been considered being much lower than other kinds of non-herbal medications.

For example, during the same period of 18 years, carmabazepine produced 380 cases of mostly severe drug eruptions. TEN, SJS, DIHS had 243 cases among them, including fatal cases. Barbital and phenobarbital produced 134 cases of drug eruption, including 63 TEN and SJS, and 4 cases were fatal in the same 18 years. Allopurinol produced 158 reported cases of drug eruption including 104 TEN, SJS and DIHS cases, among which 12 cases were fatal. Minocyclin produced 136 reported drug eruption cases including 9 DIHS and Acute generalzed erythematous pustulosis (AGEP), and 6 cases of anaphylactic shock [5].

In contrast, traditional herbal medicine consisting of mostly mixed herbs, did not show such severe adverse effects in the same period. This may be the reason why they are still permitted and used in Asian countries today.

\section{Herbal lotions could obtain good responses with severe intractable alopecia areata}

\section{Herbal lotions for alopecia areata}

Many kinds of herbs were used in China for the treatment of alopecia areata (AA) since $\mathrm{AD} 610$ when Estragali radix was recommended for the treatment $[3,4]$. During the 20th Century, the herbs in the table1 were recommended for its treatment. Among them, Laiso ${ }^{\circ}$ lotion which appeared in the late 1980s was composed of Swertia herb and Capsicum etc. to increase the blood flow of the scalp, was considerably successful to obtain response with severe intractable alopecia cases [6]. When a steamer is used on the scalp of alopecia, blood flow measured by the Doppler flow meter increased during the application of the steamer only, however, when Laiso is applied, increased blood flow maintained even after one hour of the application (Figure 1). The success of having response of hair growth was $60.8 \%, 45$ out of 74 cases of severe types of alopecia, and considering the fact that all these cases could not have any response prior to this treatment, increasing the blood flow of alopecia was considered to be effective.

Later on, an improved version called Fukisin ${ }^{\circledR}$ which also included a Tussilago farfara extract, showed better results and since then, both lotions have been put into production and used clinically (Figure 2).

Table 1. The main herbs which were recommended to create lotions for hair growth during the 20th Century (By courtesy of Professor Yu Li-ping, Beijing)

\begin{tabular}{|l|l|l|l|}
\hline No & Latin & English & Action \\
\hline 1 & Astragali radix & Astragalus root & anti-inflammatory \\
\hline 2 & Ginseng radix & Ginseng & increase blood flow \\
\hline 3 & Angelica radix & Angerica root & anti-inflammatory \\
\hline 4 & Persicae semen & Peach kernel & anti-inflammatory \\
\hline 5 & Salviae miltiorhizae radix & Danshen root & sensitization \\
\hline 6 & Zingiberis rhizome & Ginger & increase blood flow \\
\hline 7 & Cnidii rhizome & Cnidii rhizome & increase blood flow \\
\hline 8 & Carthami flos & Safflower & \\
\hline 9 & Psoraleae semen & Psoralea coryliforea fruit & \\
\hline 10 & Platyctadi cacumen & Oriental arborvitae & \\
\hline 11 & Polygoni multiflori radux & leafytwig & \\
\hline 12 & Zanthoxyli pericarpium & Zanthoxylum peel & \\
\hline 13 & Mylabris & Tiger beetle & \\
\hline 14 & Chebulae fructus & Chebula fruit & moisture retention \\
\hline 15 & Cinnamomi ramulus & Cinnamon twig & increase blood flow \\
\hline 16 & Kaempferiae rhizoma & Kaempteria root & \\
\hline 17 & Citri reticulatae pericarpaum & Gride & anti-inflammatory \\
\hline 18 & Citri grandis fructus & reticulate) & anti-inflammatory \\
\hline 19 & Saposhnikoviae radix & Citiris grandis fruit & \\
\hline 20 & Menthae herba & Saposhinikovia root & \\
\hline 21 & Rehmanniae radix & Menta herb & increase blood flow \\
\hline 22 & Camphora & Rehmannia root & \\
\hline 23 & Paeoniae radix & Camphor & \\
\hline 24 & Schizonepeta spike & Schizonepeta spike & \\
\hline 25 & Nardostachyos radix et rhizoma & Spikenard & \\
\hline 26 & Swertiae herba & Swertia herb & \\
\hline 27 & Crataegi fructus & Crataegus fruit & \\
\hline 28 & Allii satiui bulbus & Garlic & \\
\hline 29 & Glycyrrhizae radix & Licorice root & \\
\hline 30 & Asiasari radix & Flow & \\
\hline 31 & Farfarae flos & Capsici fructus & \\
\hline 32 & & & \\
\hline
\end{tabular}




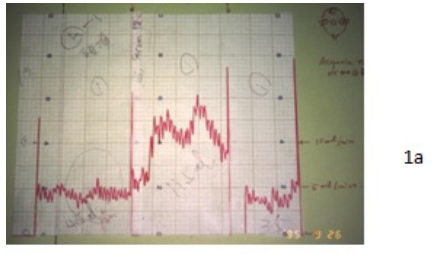

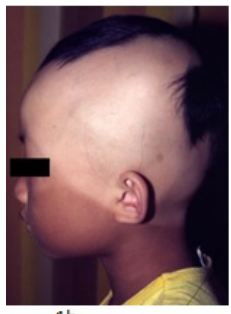

$1 \mathrm{~b}$

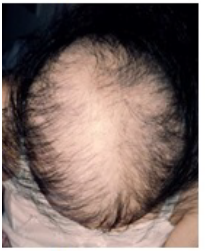

$1 d$
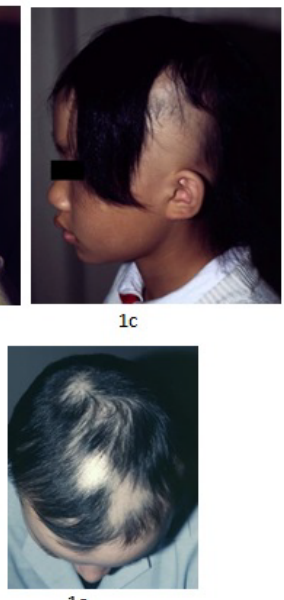

$1 \mathrm{e}$

Figure 1. Laiso forte lotion contains swertiamarin, capsaisin etc. to increase bloodflow of the scalp. When it is applied on the alopecia skin, doppler bloodflow meter indicated that bloodflow was significantly increased and maintained for 1 hour, even though a steamer was used for only 20 minutes (1a). A 9-year-old alopecia universalis (1b) could regain hair by the usage of Laiso lotion for 10 months (1c). At this age, systemic corticosteroid had to be refrained to avoid growth impairment. Another case of a 20-year-old woman who suffered from alopecia diffusa (1d) was cured by the same treatment, and her hair regrowth was satisfactory after continual usage for 11 months, without using systemic corticosteroid (1e).

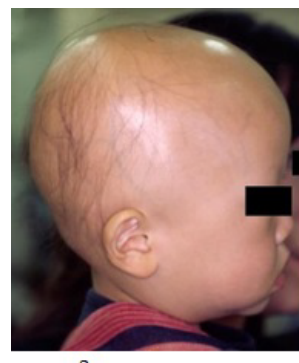

$2 a$

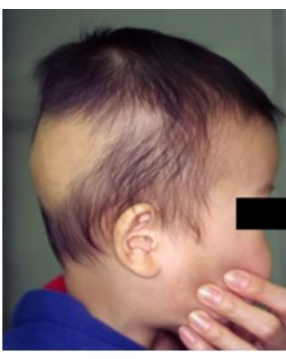

$2 \mathrm{~b}$

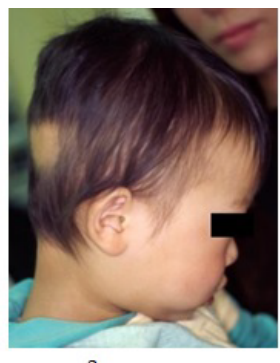

$2 \mathrm{c}$
Figure 2. 1 year-old boy who had suffered from alopecia universalis (2a) was treated only by the application of Fukisin lotion, an improved version of Laiso lotion, without using systemic corticosteroid hormone, so that the impairment of growth would never occur. Enough hairgrowth was met after 6 months (2b), and after 9 months he was cured almost completely (2c).

In 1988, Cho Zhang-gwang a Chinese pharmacologist in Beijing invented the lotion 101 for the treatment of AA. The response was tested among young Chinese army soldiers who suffered from AA of various grades of severity. As the response of hair growth proved to be higher than 90\%, he was cited and awarded with a medal in Belgium. 101 was a lotion composed of 19 herbs, and when it was applied on four cases of alopecia universalis in the author's clinic in Tokyo, all four cases responded to grow dense short hair on the whole scalp after two months of daily application, and were perfectly cured after 6 months of application without using systemic corticosteroids (Figures 3 and 4). The results were marvelous, however, soon it turned out that 101 was considerably allergic, producing eczematous eruption on the whole scalp, like Dinitrochlorbenzene (DNCB) or Squaric acid dibutyl ester (SADBE), used in western countries and the USA. Furthermore, 101 had malodor which was quite unpleasant to many patients, therefore, its usage was later abandoned in Japan.

\section{Improvement of 101}

In 1983, the author visited Dr. Cho Zhang-gwang at his factory in Beijing, and proposed to improve 101 to remove such faults, and the project began. Firstly, all the herbs which were not allowed in the regulation of herbs in Japan were removed, to leave 8 herbs which had been repeatedly used in the past 300 years in China. This newly improved lotion, AL-8, was still effective to treat severe cases of AA (table 2), and the grade of contact dermatitis was much more mild. When dermatitis happened, these 8 herbs were put into white petrolatum at $2 \%$ and $5 \%$ concentration, and patch tested on those who suffered from this dermatitis medicamentosa. The results showed that salvia miltiorrhiza radix was the contact allergen in the preparation, and at the same time, the main property of hair growth. A preparation with which salvia miltiorrhiza radix was eliminated could reproduce

Table 2. Lotion for hair growth of AL-8
\begin{tabular}{|c|c|c|}
\hline & Herbs & $\begin{array}{c}\text { amount of herbs for } \\
\text { extraction } \mathbf{( g / 3 L )}\end{array}$ \\
\hline 1 & Ginseng radix & 9.0 \\
\hline 2 & Astragali radix & 9.0 \\
\hline 3 & Angelicae radix & 8.0 \\
\hline 4 & Persicae semen & 7.0 \\
\hline 5 & Carthami flos & 3.0 \\
\hline 6 & Cnidii rhizoma & 7.0 \\
\hline 7 & Salviae miltiorrhizae radix & 6.0 \\
\hline 8 & Zingiberis rhizoma & 2.0 \\
\hline
\end{tabular}

[solvent] ethanol: purified water $=1: 1$

Herbs are immersed in the solvent for 30 days and then filtered to create a fluid for the application on the scalp.

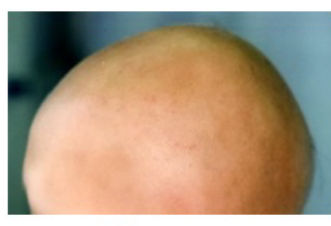

3а

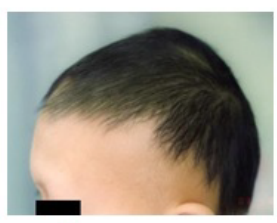

$3 b$

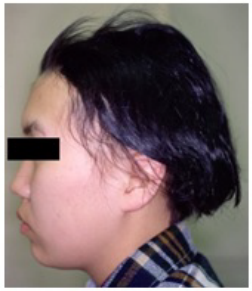

Figure 3. The hairgrowth lotion having the best performance in history was apparently 101 invented in China. All 4 cases studied in the author's clinic started to grow dense short hair after the continual application of 101 for two months. The result was really amazing, and one case (3a) is demonstrated. Note the excellent hairregrowth after 2 months (3b) and complete cure of severe alopecia after 6 months' usage (3c) without using systemic corticosteroids. Occasional production of itchy dermatitis and its odour were the defect of 101. Its response was really excellent, but still the frequent replace of severe alopecia later could not be prevented. That is why allergen control / avoidance is indispensable.

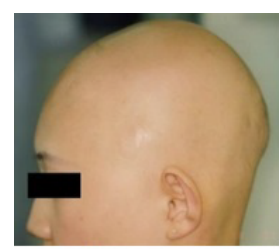

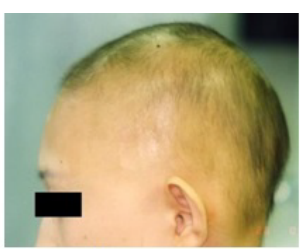

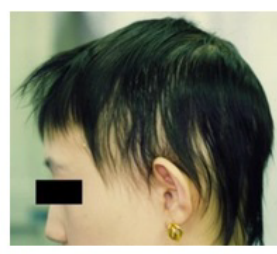

Figure 4. Another case of alopecia totalis which had been present for nearly two years on a 21 -year-old woman (4a) responded excellently to the application of 101 without using systemic corticosteroids. After 3 months dense short hair grew (4b), and 9 months of usage brought complete cure (4c). 101 was certainly the best hairgrowing lotion, and it was regrettable that it was allergenic and had mal odour. 
hair, but, the rate of its effectiveness was lowered by $10 \%$, and the speed of hair growth was much slower. Therefore, in order to obtain response of hair growth, salvia miltiorrhiza radix should not be eliminated, even though it was mildly allergic when its concentration was lowered for safety. Such improved lotions were not considered to be of the 101 family in Beijing already at that time.

Today, a simpler herb lotion, AL-4 (Table 3), has been used for the treatment, leaving only the indispensable herb components. The results of response was almost comparable to its predecessor AL-8 (Figure 5) and AL- 4 can be made by any pharmacy in a city that has the ability of making such lotions by immersing herbs in ethanol for a month, and filter the contents to leave herb lotion for the application.

When mild dermatitis appears, the usage of AL-4 is halted to be replaced by the more mild Laiso ${ }^{\star}$ or Fukisin ${ }^{\star}$ which does not contain salvia miltiorrhiza radix, while still continuing to be as effective as possible in hair regrowth.

\section{How to prevent hair loss, after successful hair regrowth Relapse should be prevented}

Severe AA cases have two difficult problems. The first one is the difficulties of hair regrowth, and the second one is that regrown hair is suddenly or gradually lost from the patients and the original severe AA conditions come back. The latter is really a nightmare for both the patients and doctors.

Alopecia areata (AA) becomes highly problematic to cure when it develops to severe types, such as A. diffusa, A. reticularis, A. totalis and A. universalis. This is because firstly, severe alopecia does not respond easily to antisymptomatic treatments, and secondly the regrown hair is suddenly or gradually lost to go back to the previous severe alopecia, even when antisymptomatic treatment is being continued (Figures 6 and 7).

The causation of severe AA turned out to have been an allergic reaction as it was temporarily cured by the usage of perorally

Table 3. The effect of AL-8 on alopecia areata (Years: 1990-1995).

1. First group (Mild cases of alopecia areata which did not require systemic corticosteroid theray)

Cases: $\mathrm{n}=22$ ( 7 males and 15 females)

Mean age: 39.0 (1-78)

(Alopecia universalis

Alopecia diffusa

Alopecia areata multiplex confluents

Alopecia areata multiplex

Alopecia areata (single lesion)

2
1
1
12
6

5
3
1
2
7

Cemarkable improvement (including cure)

( Improvement

Slight improvement

No effect

Drop out

Rate of effect:

$72.7 \%$

Worsening factors
1. ANA
2. Serum elevated $\operatorname{IgE}$
3. Metal allergy

$\begin{array}{ccrc}\text { Positive } & \text { negative } & \text { NT } & \text { Total } \\ 8 & 9 & 5 & 22 \\ 9 & 11 & 2 & 22 \\ 14 & 2 & 6 & 22\end{array}$

2. Second group (severe cases which required combination therapy of systemic corticosteroid hormones : Pulse therapy of perorally administered prednisolone at $10 \mathrm{mg}$ for a week, then $5 \mathrm{mg}$ for a week and $0 \mathrm{mg}$ for two weeks, repeated every month, or one shot injection of triamcinolone acetonide at $50 \mathrm{mg}$, repeated once a month) Cases: $\mathrm{n}=40$ (10 males and 30 females)

Mean age: $31.1(8-72)$

Alopecia universalis

Alopecia totalis

Alopecia diffusa

Alopecia areata multiplex confluents

Alopecia areata (multiplex)

Effect of applying AL-8 twice a day along with systemic usage of corticosteroid hormones

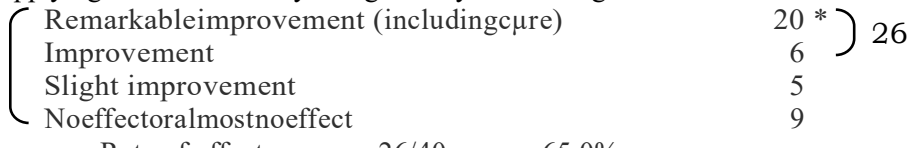

$\begin{array}{lll}\text { Rate of effect: } & 26 / 40 & 65.0 \% \\ \text { * Recurrence rate: } & 13 / 20 & 65.0 \%\end{array}$

Worsening factors

1. ANA
2. Serum elevated IgE
3. Metal allergy

$\begin{array}{ccrr}\text { Positive } & \text { negative } & \text { NT } & \text { Total } \\ 9 & 31 & 0 & 40 \\ 19 & 21 & 0 & 40 \\ 22 & 18 & 0 & 40\end{array}$




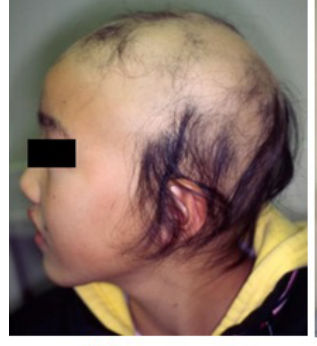

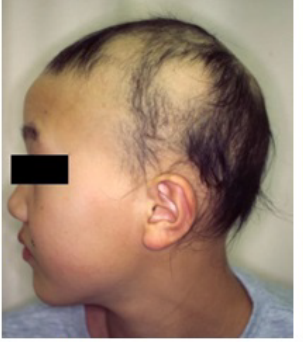

$5 b$
$5 c$

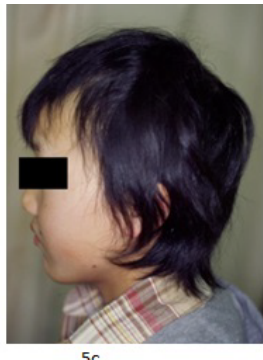

Figure 5. AL-8, an improved hairgrowing lotion having 8 herbs, instead of 101 's 19 herbs, could regrow hair all right without using systemic administration or injection of corticosteroids. An 11-year-old female repeated hairloss for the past few years (5a) began applying AL-8 everyday. Her hair regrew densely after 7 months (5b), and complete cure was noted a year later $(5 \mathrm{c})$. Hair was retained for a year and half later. The response was slower than 101, however, complete cure was obtained.

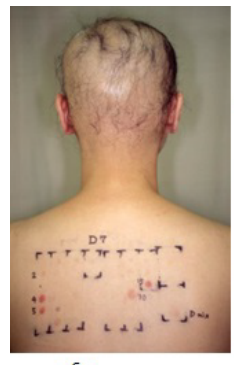

$6 a$

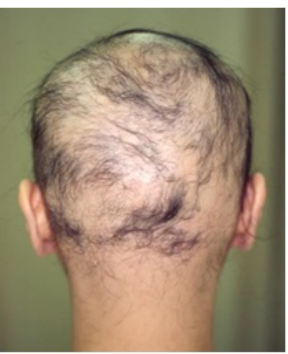

$6 \mathrm{~d}$

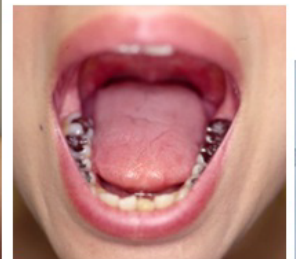

$6 \mathrm{~b}$

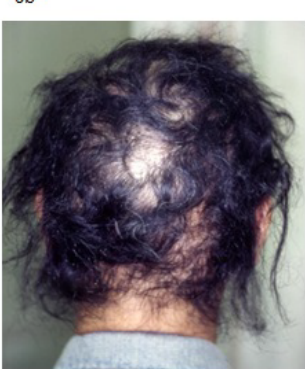

$6 e$

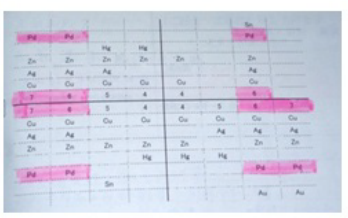

$6 c$
Figure 6. As relapse of severe alopecia is quite common ( $>50 \%)$, the removal of allergens that break IP is necessary along with antisymptomatic treatment. A 35-year-old female having alopecia universalis for the past 3 years (6a) turned out to be sensitized by nickel and palladium. Analysis showed that there were 7 dental metals which contained palladium ( $6 \mathrm{~b}$ and $6 \mathrm{c}$ ), and their removal assisted in the regrowth of hair in 5 months (6d), and almos complete cure 11 months later (6e). There was no relapse of this severe alopecia thereafter.

administered prednisolone (PSL) [7,8], or intramuscular injection of triamcinolone acetonide (TCA) once a month $[9,10]$. Also, a biopsy of AA revealed that hairfollicles were destroyed by the invasion of mainly CD4 T lymphocytes, mixed with a smaller number of CD8 T lymphocytes. Hair follicles are composed of keratin which is also a main component of epidermal cells. As keratin is the most important superficial component to assure the strength of skin and hair from the physical forces outside of the body, normally $\mathrm{T}$ lymphocytes do not attack it, recognizing keratin as a self-component. Therefore, when it turned out that in AA T lymphocytes attacked hair follicles composed of keratin, the interpretation for this self-damaging phenomenon to result in defluvium was observed to be a failure of immune privilege (IP) [11]. Most suspected mechanism to produce such failure of IP has been autoimmunity, even though evidence for it has been obscure. Certainly, serum antinucleic antibody (ANA) of severe AA turned out to have been positive at $31.0 \%$ (9 out of 29), however, the positive cases at serum diluted 160 times was only 3 cases (10.3\%) [6]. Other cases showed positive ANA at serum diluted 40-80 times. This test was performed by the SRL Company in Tokyo, and it was reported that $6.8 \%$ out of 1,000 persons containing all sorts of diseases showed positive ANA, and that $64(10.5 \%)$ out of 593 female patients whose diseases were not autoimmune diseases showed positive ANA at 4080 times serum dilutions. The same positive rates with men without autoimmune diseases were 5 (1.2\%) out of 411 . This fact meant that with females, when the ANA positive rates are more than 160 times of serum dilution, it can be diagnosed as autoimmune disease. With the obtained data showing a positive rate of $10.3 \%$ among AA cases, autoimmunity can be considered as a causation of IP failure, but it cannot be the main causation as the remaining $90 \%$ of cases of AA did not show positive for autoimmunity. Animal experiments featuring implanting human scalp components on rats or mice, could successfully produce alopecia, however, the T lymphocytes which destroyed these animal hairfollicles were CD8 and NKG2D positive cells [11], therefore, the composition was different from human AA. Also, human hairfollicle keratin used for the experiment cannot be said to be autoimmunity for these such animals (Tables 4 and 5).

Therefore, apart from autoimmunity, hapten allergies and atopic allergies have been investigated as causations since the 21st century. This is because when natural keratin were altered by conjugating

Table 4. Lotion for hair growth of AL-4

\begin{tabular}{|c|c|c|}
\hline & Herbs & $\begin{array}{c}\text { amount of herbs for } \\
\text { extraction }(\mathrm{g} / 60 \mathrm{ml})\end{array}$ \\
\hline 1 & Ginseng radix & 5.4 \\
\hline 2 & Astragali radix & 5.4 \\
\hline 3 & Angelicae radix & 4.2 \\
\hline 4 & Salviae miltiorrhizae radix & 3.6 \\
\hline 5 & Ethanol & $33.0 \mathrm{ml}$ \\
\hline
\end{tabular}

Herbs are immersed in the solvent for 30 days and then filtered to create a fluid for the application on the scalp.

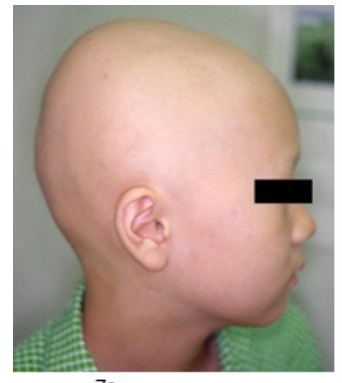

$7 a$

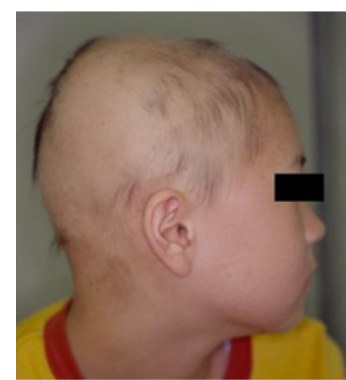

7c

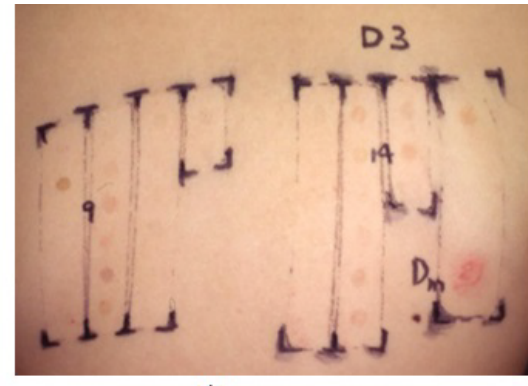

$7 \mathrm{~b}$

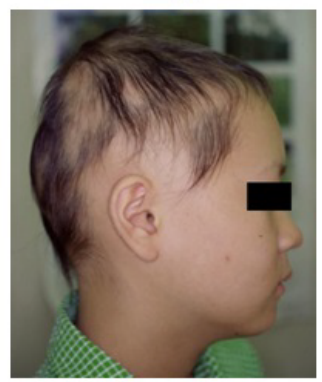

$7 d$
Figure 7. With atopic alopecia, as its main causation is house dust mite (HDM) allergy, HDM should be counted in the patients' home, so that an environmental improvement could reduce HDM to less than $50 / \mathrm{m} 2 /$ seconds aspiration with a $320 \mathrm{~W}$ vacuum cleaner. A 9-yearold girl suffering from alopecia universal is since the age of 5 (7a) showed clear allergic reaction to HDM by a patch test (7b). To prevent relapse, this environmental improvement was performed along with the application of AL-8 lotion, which regrew hair in 9 months (7c), and prevented relapse for one year and 3 months $(7 d)$. 
Table 5. The rate of satisfactory improvement of severe alopecia areata in which over 70\% of hair regrowth on the scalp was met by the usage of AL-4 lotion.(Years $1996-2015)$

1. metal hypersensitive alopecia areata

Patch test positive to metals

$$
\begin{aligned}
& \frac{102}{146}=69 \cdot 9 \% \\
& \frac{37}{49}=75 \cdot 5 \%
\end{aligned}
$$

Rate of improvement after elimination of metals from oral cavity and or pans

(Cure continued for 2 to 12 years, on average of 4 years 4 months)

2. Atopic alopecia

serum $\operatorname{IgE}>250 \mathrm{IU} / \mathrm{ml}$

$\frac{29}{64}=45 \cdot 3 \%$

Rate of improvement after environmental improvement

3. Alopecia presumably due to autoimmunity

serum ANA positive

(at 1:160 1:320 dilution)*

$\frac{1}{70}=1 \cdot 4 \%$

Rate of improvement only by antisymptomatic treatment

* Serum ANA positive at 1:80 dilution or less turned out to be within normal female limit.

haptens or atopic allergens there is enough evidence that IP is surely broken. One of such haptens were metal ions which are elements therefore unbreakable in the body and have strong affinity to keratin $[12,13,14]$. Allergic contact dermatitis due to metal ions have been very well known in the world, and dermatitis could be provoked not only by contact to skin, but also by perorally administered metal ions, which were absorbed from the intestine and reached the skin via blood flow from inside. Many such cases were previously reported and published [15].

\section{Mite allergy in atopic alopecia}

Other allergens investigated were house dust mite (HDM) components which were the major causative allergens to produce severe cases of atopic dermatitis (AD) [15]. AD had been known to produce AA since the 1970s, having been reported as "Atopic alopecia" by several authors $[16,17]$. Previously, the causation of AD had not been known. However, after a national research project on $\mathrm{AD}$ had been performed for three years from 1989 by three hospitals in Japan, evidence was accumulated that the main causation of $\mathrm{AD}$ was no doubt HDM. A new method known as the Methylene blue agar (MBA) method, to clarify the mite number, mite fauna, in the patients' homes, enabled to perform an environmental improvement to decrease the mite number to less than $50 / \mathrm{m} 2 / 20$ seconds aspiration by a $320 \mathrm{~W}$ electric cleaner. This technique enabled to decrease the mite number at everywhere in the home to less than $1 \mathrm{mite} / 10 \mathrm{~cm} \times 10 \mathrm{~cm}$.

According to the patch test results using crashed live mites on plastic discs of $8 \mathrm{~mm}$ in diameter, it proved not to produce eczematous reactions by HDM [18].

As HDMs are invisible because of their size of $0.3 \mathrm{~mm}$, their presence is not recognized by the inhabitants. After the MBA method was executed to inform $\mathrm{AD}$ patients of how many HDMs were present in the interior of their houses, an environmental improvement to attain less than $50 / \mathrm{m} 2 / 20$ seconds aspiration was realized. A double blind test (DBT) was performed and it assured remarkable improvement for highly severe skin conditions for a long period $[18,19]$. The main technique for environmental improvement is the removal of carpets and tatami mattresses to be replaced by flooring or heater flooring and introducing mite-free mattresses and pillows. The surfaces of sofa and chairs had to be replaced by artificial leather to remove stimulating living conditions for HDMs. Tan et al also reported a favorable effect of environmental improvement by DBT measuring Der P instead of MBA [21]. Such techniques of environmental improvement was also applied to severe intractable atopic alopecia to stop the recrudescence of alopecia after the antisympromatic treatment regrew the patients' hair, and was followed up for more than two years.

\section{Conclusions}

There has been a long history of usage of herbs especially in China, and some herbal lotion could regrow hair successfully in alopecia areata. With alopecia areata immune privilege (IP) has been considered to be broken to result in the destruction of hair follicles, and recently there were two trigger factors to break IP: metal hypersensitivity and mite allergy of atopic dermatitis. In treating alopecia areata, prevention of relapse is as important as the antisymptomatic treatment composed of temporal systemic usage of corticosteroid and application of herb lotions. For that purpose, metal allergy and mite allergy should be investigated followed by the necessary elimination of causative metal ions from the oral cavities and pans, or mite fauna investigation of the patients' homes for the environmental improvement. Autoimmunity should be still continually investigated.

\section{References}

1. Kitazima MK (2017) Science Visual Review, Life Science, Tokyo.

2. Ogawa T: Medical History, Chuko-Shinsho No.39, 1964, 3-234, Chuoh-Koron, Tokyo.

3. Zhin Do Sei: Reprint of Zhu Pying Eng Hoh Rung I, Renmin Sanitary Publication Beijing, 2000: 1-754

4. ibid II, 755-1420

5. Fukuda E, Fukuda H (2015) Drug Eruption Information from 1980 to 2014, 16th Ed, Fukuda Hifuka Clinic, Shimen Chuo, Fukuoka prefecture 347-353.

6. Koizumi M, Nakayama H, Ebihara T (1998) Laboratory test results and the effect of treatment in 106 cases of intractable alopecia areata, Jap J Dermatol 108: 1881-1891. 
7. Kern F, Hoffman WH, Hambrick GW, Blizzard RM (1973) Alopecia areata. Immunologic studies and treatment with prednisone. Arch Dermatol 107: 407-412

8. Winter RJ, Kern F, Blizzrd RM (1976) Predonisolone therapy for alopecia areata. Arch Dermatol 112: 1549-1552

9. Michalowski R, Kuczynska L (1978) Long-term intramuscular triamcinolon-acetonide therapy in alopecia areata totalis and universalis. Arch Dermatol Res 261: 73-76

10. Kurosawa M, Nakagawa S, Mizuashi M, Sasaki Y, Kawamura M, et al. (2006) A comparison of the efficacy, relapse rate and side effects among three modalities of systemic corticosteroid therapy for alopecia areata. Dermatology 212: 361-365.

11. Guo H, Cheng Y, Shapiro J, McElwee K (2015) The role of lymphocytes in the development and treatment of alopecia areata. Expert Rev Clin Immunol 11: 13311351 .

12. Nakayama H (2002) New Aspects of Metal Allergy. Acta Dermatovenereol 10: 207 219

13. Nakayama H (2006) Dental Metal Allergy. J Environ Dermat 13: 57-65

14. Nakayama H (2012) Metal hypersensitive alopecia -A new disease with metal allergy. Clinical Allergy 32: 697-702.
15. Nakayama H, Chen KR (2017) The remarkable effects of allergen control on various chronic intractable allergic diseases, such as pigmented cosmetic dermatitis, chemical induced SLE, pustulosis palmaris et plantaris, atopic dermatitis and severe cases of alopecia areata, $J$ Environ Dermat.

16. Ikeda T (1965) A new classification of alopecia areata, Dermatologica 131: 421-445

17. Katsuoka K, Arai A, Kameyama K, Noguchi T, Nishiyama S (1992) Atopic alopecia areata, Hifubyo-Shinryo. Dermatosis Treatment 14:729-733.

18. Nakayama H (1995) The role of the house dust mite in atopic eczema In: Practical Contact Dermatitis, McGraw-Hill, NY pp: 623-630

19. Kumei A (1995) Investigation of mites in the houses of atopic dermatitis (AD) patients, and clinical improvements by mite elimination. Arerugi 44: 116-127. [Crossref]

20. Kumei A, Nakayama H, Sakurai M, Tsurumachi K, Takaoka M (1990) [Results of patch test using mite components in atopic dermatitis (AD) patients. 1st report. "As is patch test" using crushed mites]. Nihon Hifuka Gakkai Zasshi 100: 1127-1134. [Crossref]

21. Tan BB, Weald D, Strickland I, Friedmann PS (1996) Double-blind controlled trial of effect of housedust-mite allergen avoidance on atopic dermatitis. Lancet 347: 15-18. [Crossref]

Copyright: @2017 Nakayama H. This is an open-access article distributed under the terms of the Creative Commons Attribution License, which permits unrestricted use, distribution, and reproduction in any medium, provided the original author and source are credited. 